Proceeding Paper

\title{
Location-Assistive and Real-Time Query IoT-Based Transport System ${ }^{\dagger}$
}

\author{
Saqlain Abid ${ }^{1, *}$, Muhammad Jehanzeb Irshad ${ }^{1}$, Muhammad Wasif ${ }^{1}{ }^{\circledR}$, Zubair Mehmood ${ }^{1}$, Iftikhar Hussain ${ }^{1}$ \\ and Muhmmad Hassan Ejaz ${ }^{2}$
}

1 Electrical Engineering Department, University of Gujrat, Gujrat 50700, Pakistan; jehanzeb.irshad@uog.edu.pk (M.J.I.); syed.wasif@uog.edu.pk (M.W.); zubair.mehmood@uog.edu.pk (Z.M.); iftikhar.hussain@uog.edu.pk (I.H.)

2 Electrical Engineering Department, Federal Urdu University, Islamabad 44000, Pakistan; hassanchahil64@gmail.com

* Correspondence: saqlainabid111@gmail.com

+ Presented at the 1st International Conference on Energy, Power and Environment, Gujrat, Pakistan, 11-12 November 2021.

Citation: Abid, S.; Irshad, M.J.; Wasif, M.; Mehmood, Z.; Hussain, I.; Ejaz,

M.H. Location-Assistive and

Real-Time Query IoT-Based

Transport System. Eng. Proc. 2021, 12, 101. https://doi.org/10.3390/ engproc2021012101

Academic Editor: Qasim Awais

Published: 23 February 2022

Publisher's Note: MDPI stays neutral with regard to jurisdictional claims in published maps and institutional affiliations.

Copyright: (C) 2022 by the authors. Licensee MDPI, Basel, Switzerland. This article is an open access article distributed under the terms and conditions of the Creative Commons Attribution (CC BY) license (https:// creativecommons.org/licenses/by/ $4.0 /)$.

\begin{abstract}
In this age, the automotive surveillance service is a well-known proven product implemented in conventional transport systems and widely known vehicle entities worldwide. It is a system that can be very robust and reliable. Through this work, GPS (Global Positioning System is implemented, which is used to locate and identify any automobile. This concept is an integrated device that can steadily track a speeding object and communicate the device's status whenever its identification is required. It must obtain the NodeMCU (ESP8066 module) for the network connection device, which is routinely connected to the GPS device and the Arduino board through which it functions. The NodeMCU is used to relay the device's status simultaneously from a distant location. The context of the application, "Bus Management System", which is designed in MIT, provided an android studio for researchers to design any application interface. The network interface could be reviewed on the accounts-receivable public network in the form of charts. The program shows responses to the mobile application; it only requires logging in with a data network. The application, "Bus Management System", displays the sensor values in terms of GPS coordinates statistics. This IoT based transport assistive system is cost effective and less complex from other existing IoT based transport assistive system.
\end{abstract}

Keywords: GPS (Global Positioning System); NodMCU; ESP8066 module

\section{Introduction}

The curiosity towards the smart bus system is just a minor part of an intelligent transport system. As of now, many small applications have been used such as track and trace and anti-theft smart response systems. Current technologies mostly use GSM/GPRS technologies, which are outdated and restricted due to their uncontrolled consequences caused by many misconducts and heinous crime offenses, such as remote-control, signalattached bombs. These GSM (Global System for Mobile)-based technologies create a prompt message with the help of GPS, the acquired longitude and latitude of the current location is sent to the other end user, and then, with these values and using Google Maps, the exact location can be seen even if the bus has been stolen [1]. RFID (Radio Frequency Identification) has short-range, max-band rates of data. It uses the communication medium between the bus and the bus station. Both end points have modules: one of them acts as the TX node or the transmitting node and the other acts as the RX or receiver node. As compared to other mediums during the modern era, a smart system for fleet management is the need of the hour. The current technology of track and trace is used, and the programmable data 
is run on the map accordingly. The proposed project using GPS and RF modules is more economical as compared to other existing methods [2].

\section{Literature Review}

In the past decade, the world has changed its approach towards the growing economy. The economists, in the latest research, studied the large impact of minor systems on the main-state economy. The public transport systems in any country are in the mainstream of revenue generation for the country. The public transporting companies with extensive facilities enjoy a successful profit margin too. However, the public interest is now diverse because of the influence of modern technology. The customer shows reluctance in use of these outdated transport mediums; therefore, by keeping an eye on customers' needs, transport companies have started to work on "smart bus management systems" [3].

There is a tracking system for users of public transport systems, which, with the help of the wireless technology called the "RFID (TAG)", is robust and accurate. This is not only a presentable technique of data transformation but also provides a better substitute in those areas where no other medium is applicable and insufficient reliability is provided. The proposed subject was used in inter-building or under the same roof applications. The next level to these systems is the inter-communication between the vehicle and its destination. The use of an RFID here will drastically lower the cost; you do not need any other controller, simply an RFID module. RFID is the most important network and low-power operating device, as compared to Wi-Fi and Wi-max, especially in those regions where no electricity is available. Generally, the GPS traces the longitude and longitude with the locality of the bus system. This information is stored as data on the IoT open platform [4-6].

\subsection{Autonomous Automobile Location (AAL) System}

An AAL system is an advanced method providing the route and a complete package of any third party involved in the remote location of the vehicle, with the help of guided GPS signals. AAL encompasses a Global Positioning System (GPS) and Earthly Indication System (EIS) to provide the actual physical location of the vehicle. An AAL system involves PC-based aggravation software for lettering, a wireless system, GPS handset on the automobile, and GPS satellite spreading. Between the two forms of AAL, GPS-based and signpost-based, the GPS-based system is extensively cast off [7].

\subsection{Global Positioning Associated Systems (GPAS)}

With unassisted GPS, locating the satellites, receiving the information, and identifying a precise location may take several minutes. The tracking practice of GPAS uses GPS satellites to identify the roads that vehicles use. A GPS receiver in a vehicle is always in communication with four satellites (three satellites regulate latitude, longitude, and the fourth provides an element of time); hence, it is always able to accurately locate a vehicle. The location of the bus is narrowed down to a range of between $3 \mathrm{~m}$ and $8 \mathrm{~m}$, over a distance of $1 \mathrm{~km}$ using this technique. Evidence such as vehicle location, average speed, direction, path spanned in a nominated period, and alerts are taken by the tracking system to the unfitting station.

\subsection{Radio Frequency Based Identification TAGS (RFID)}

Reverse logistics is an empathic identity mechanism that uses marks deployed by users to retrieve information dynamically. To retrieve data from marks, device systems use radio communication. The automotive proof of this is shown to a specific RFID student by the RFID tag that holds electricity and electronics, which is then transmitted via an application. The location of the vehicle is distributed by this method with consistency from $4 \mathrm{~m}$ to $6 \mathrm{~m}$ [8]. 


\section{Research Methodology}

The automobile pursuing system is a widely used system, which is a more proficient and reputable modern technology. This system is adaptable for all the reputed companies at present, as it is not that difficult to place anywhere on the vehicle, less costly, and more efficient. In this designed project, the route of the specific bus is tracked in real-time with the assistance of a global positioning system. The primary procedure is that the data strings attached to the module are shifted in real-time via NodMCU (Wi-Fi module), fetching an open-source code that is provided by a third party. To implement the design of the project, the hardware components of MCU are connected to the controller and the GPS receiver. The purpose of this is to stream the data continuously on the webpage via real-time data strings. The total additional data are bus speed and station location with longitude and latitude.

Whenever a request is made through the channel, the resultant graphs with real-time tracking show up in channel fields. The system of "FIREBASE" automatically shows a return reply to the mobile application; it simply requires to add a specific Channel ID. The app, FIREBASE, accurately indicates the position.

\subsection{Hardware Components Used}

- $\quad$ GPS module;

- $\mathrm{RF}(\mathrm{AC}-11)$;

- ESP8266 WiFi Module;

- Arduino UNO;

- IR Sensors.

\subsection{Software Interfaces Used}

- $\quad$ Firebase Channel Field;

- $\quad$ MIT APP (Bus Management System);

- Android Studio.

By using the FIREBASE server, it needs to be first configured the account with desired fields. The number of fields required could be increased or decreased; however, these only used two fields because we required only the longitude and the latitude. After a time, delay, the GPS + ESP8266 sends the current values of the longitude and latitude field, which were previously configured to obtain the values.

The configuration of these fields might require an API key-generated field for reading the data and writing on the FIREBASE server. Here, we used the writing API key, which allowed the data to push the values and generate the graph in the required fields.

For the graph of the values, the field might be delayed by up to $10 \mathrm{~s}$, after which the page must be refreshed. It also shows the time at which the values have been posted.

\section{Results and Conclusions}

The website and the designed application (Bus Management System) display the final performance showing the position of the shuttle, the three domains generated on the program called the magnitude of the prime meridian, and the bus numbers including their respective bus stations. Using the markup and website server (FIREBASE) API (Application Program Interface), which was linked through the public network on FIREBASE, we most likely built this link. This synchronization requires a certain setup, such as the literacy of the client API keys, but allows a person to access your connection data and process anything regarding a client, so a reference voltage can be obtained to determine the feature vector and this information can be published on the website. Today, fleet management uses tracking protocols and assets protection by tracking with the help of a GPS and NodeMCU that uploads the tracker data to a specific server in real-time. To upload the data stream, a controller is also used to provide the data stream with two types of code instruction. The first type sends the data while the other type fetches the code that obtains the data stream, which is then uploaded to the server. The server used here is an open-source code IoT 
(Internet of Things) platform named FIREBASE. It not only uploads the data stream but is also able to draw a chart as an easily viewable graph. At this open-source cloud, we can make only eight different types of fields and channels up to a certain level. Data limits exist and only a certain KB can be uploaded free of charge. The RF (radio frequency) modules used here for a certain type of bus station show the arrival of the bus at a specific station.

Table 1 shows the delay time values when refreshing the values in real-time. The main purpose of the proposed project is to provide tracking with a new interface and maximum features, keeping all the gadgets in one channel and on one page with real-time data streams that are uploaded instinctively. This IoT based transport system is cost effective and less complex from other existing IoT based transport assistive system.

Table 1. Delay time values when refreshing the values in real-time.

\begin{tabular}{cccc}
\hline Field 1 (Longitude) & Field 2 (Latitude) & Bus Station & Delay (s) \\
\hline 33.741 & 74.206 & 1 & $9 \mathrm{~s}$ \\
33.832 & 74.560 & 2 & $6 \mathrm{~s}$ \\
33.982 & 74.890 & 3 & $7 \mathrm{~s}$ \\
\hline
\end{tabular}

Author Contributions: Conceptualization, S.A.; methodology, S.A.; software, M.H.E.; validation, I.H. and Z.M.; writing—original draft preparation, S.A.; writing—review and editing, M.J.I. and M.W. All authors have read and agreed to the published version of the manuscript.

Funding: This research received no external funding.

Data Availability Statement: Not applicable.

Conflicts of Interest: The authors declare no conflict of interest.

\section{References}

1. Bajaj, D.; Gupta, N. GPS based automatic vehicle tracking using RFID. Int. J. Eng. Innov. Technol. 2012, 1, 31-35.

2. Chattaraj, A.; Bansal, S.; Chandra, A. An intelligent traffic control system using RFID. IEEE Potentials 2009, 28, 40-43. [CrossRef]

3. Arebey, M.; Hannan, M.A.; Basri, H.; Abdullah, H. Solid waste monitoring and management using RFID, GIS and GSM. In Proceedings of the 2009 IEEE Student Conference on Research and Development (SCOReD), Serdang, Malaysia, 16-18 November 2009.

4. Al-Ali, A.; Aloul, F.A.; Aji, N.R.; Al-Zarouni, A.A.; Fakhro, N.H. Mobile RFID tracking system. In Proceedings of the 2008 3rd International Conference on Information and Communication Technologies: From Theory to Applications, Damascus, Syria, 7-11 April 2008.

5. Zhao, H.; Irshad, M.J.; Shi, H.; Xu, W. Passive source localization using compressive sensing. Sensors 2019, 19, 4522. [CrossRef] [PubMed]

6. Ali, U.; Muhammad, W.; Irshad, M.J.; Manzoor, S. Multi-sensor fusion for underwater robot self-localization using PC/BC-DIM neural network. Sens. Rev. 2021. [CrossRef]

7. Weston, D.C.; Barney, J.A. Method of Game Play Using RFID Tracking Device. U.S. Patent 6,761,637, 13 July 2004.

8. Pandit, A.A.; Mundra, A.K.; Talreja, J. RFID tracking system for vehicles (RTSV). In Proceedings of the 2009 First International Conference on Computational Intelligence, Communication Systems and Networks, Indore, India, 23-25 July 2009. 